\title{
ESTUDO COLORIMETRICO DA MONTMORILLONITA II. INFLUENCIA DA ELIMINAÇÃO DOS ÓXIDOS DE FERRO LIVRES NA REAÇÃO DA MONTMORILLONITA COM A BENZIDINA
}

\author{
Wanderley José de Mello \\ antonio Carlos Teixeira Mendes
}

\section{RESUMO}

Este trabalho teve por objetivo estudar a influência da eliminação ou não dos óxidos de ferro livres na reação da montmorillonita com a benzidina, assim como dos métodos usados com tal finalidade. Para tal, suspensões de cinco (5) tipos de montmorillonita, sendo uma de procedência desconhecida $(\mathrm{Mx})$ e quatro descritas pelo Projeto 49 (M22, M20, M22a e M31), foram submetidas a três tipos de tratamento:

a) Eliminação dos sais solúveis, catíons divalentes trocáveis, matéria orgânica e óxido de manganês livre;

b) Mesmo tratamento citado em a e mais a eliminação dos óxidos de ferro livres pelo método do H-nascente

c) Mesmo tratamento citado em a e mais a remoção dos óxidos de ferro livres pelo método do bicarbonato - citrato - ditionito de sódio.

Os resultados indicaram que houve influência do tipo de tratamento em todas as montmorillonitas testadas, sendo $o$ teste $F$ significativo ao nível de $1 \%$.

A conclusão geral a que se pôde chegar foi que a eliminação dos óxidos de ferro livres parece concorrer para maior uniformização da cor azul obtida, sendo que o método do $\mathrm{H}$-nascente apresentou os melhores resultados, não apenas no que se refere à uniformização, mas também quanto à intensidade da cor obtida.

\section{INTRODUÇÃO}

Quando se pensa em fazer uso da reação da benzidina com a motmorillonita com a finalidade de se estabelecer um método quantitativo para a determinação deste mineral de argila, um problema vem logo à mente: deve-se ou não proceder à eliminação dos óxidos de ferro livres? Qual o método mais indicado para esta remoção?

* Entregue para publicação em 13/7/1972

** Aluno do Curso de Pós-Graduação em Solos e Nutrição de Plantas da ESALQ e bolsista da FAPESP.

*** Professor de Disciplina do Departamento de Solos e Geologia da ESALQ. 
HENDRICKS e ALEXANDER (1940) admitiram que, provavelmente, a oxidação da benzidina pela montnorillonita se devia à presença do ferro férrico, sendo que o ferro ferroso impedia o desenvolvimento de semiquinonas. Por outro lado, a cor vermelha dos solos mascarava a cor azul da reação, tornando-a verde ou azulesverdeada.

PAGE (1941) mencionou que a eliminação dos óxidos de ferro livres dos colóides do solo ou de bentonitas destruia o poder dos mesmos de reagir com a benzidina.

TEIXEIRA MENDES e MELLO (não publicado) observaram que a montmorillonita 22 (amarela devido à presença de óxidos de ferro livres) reagia com uma solução aquosa de benzidina com velocidade muito maior que uma montmorillonita de procedência desconhecida (branca, aparentemente isenta de tais óxidos). Porém, uma vez procedida a eliminação dos óxidos de ferro livres da montmorillonita 22 , sua velocidade de reação diminuia, assemelhando-se à montmorillonita de procedência desconhecida neste mistér.

Segundo SIEGEL (1945) montmorillonitas que não reagiram com a benzidina dariam a cor esperada se algumas gotas de uma solução diluída de cloreto de ferro $\left[\mathrm{Fe} \mathrm{Cl}_{3}\right]$ fossem adicionadas.

WEIL - MALHERB e WEISS (1948) chegaram à conclusão de que a reação da montmorillonita com a benzidina é uma oxi-redução, sendo que um pré-tratamento destes minerais inibia a reação. A oxidação de aminas aromáticas seria devida a ions metálicos da rede cristalina.

GRIM (1953) admitiu que a aplicação dos métodos de coloração para a identificação dos minerais de argila era restrita, sendo um dos fatores limitantes, o mascaramento da cor devido à presença dos óxidos de ferro.

A luz destas informações, chegou-se à conclusão de que se deveria eliminar os óxidos de ferro livres, visando-se a :

a) uniformizar a velocidade da reação

b) caso seja realmente o ferro férrico o responsável pela oxidação da benzidina, eliminar as diferenças que ocorrem nos vários tipos de solo;

c) aumentar a pureza da cor obtida na reação.

Assim sendo, este trabalho estuda a influência de três tratamentos na reação da montmorillonita com a benzidina: um, onde os materiais em estudo sofreram tratamento para a eliminação dos 
sais solúveis, catíons divalentes trocáveis, matéria orgânica e óxido de manganês livre, e outros dois tais que, além destes tratamentos, tiveram também seus óxidos de ferro livres eliminados, ora pelo método de H-nascente (JEFFRIES e JACKSON, 1949), ora pelo método do bicarbonato - citrato - ditionito de sódio (AGUILLERA e JACKSON, 1953; MEHRA e JACKSON, 1960).

\section{MATERIAL E MÉTODO}

\section{1 - Material}

\section{1 - MINERAIS PADRÕES}

- Suspensão de $\mathrm{Mx}$ a $50 \mathrm{ug} / \mathrm{ml}$ e com tratamentos para eliminação dos sais solúveis, catíons divalentes trocáveis, matéria orgânica e óxido de manganês livre.

- Suspensão de $\mathrm{Mx}$ a $50 \mathrm{ug} / \mathrm{ml}$ com os mesmos tratamentos acima e mais a eliminação dos óxidos de ferro livres pelo método do H-nascente.

- Suspensão de $\mathrm{Mx}$ a $50 \mathrm{ug} / \mathrm{ml}$ e com tratamentos para eliminação dos sais solúveis, catíons divalentes trocáveis, matéria orgânica, óxido de manganês livre e óxidos de ferro livres, estes pelo método do bicarbonato-citrato-ditionito de sódio.

- Suspensões de M22, M20, M22a e M31 a $50 \mathrm{ug} / \mathrm{ml}$ e com os mesmos tratamentos indicados para a $\mathrm{Mx}$.

\section{2 - APARELHOS}

- Centrífuga Internacional n. ${ }^{\circ} 2$.

- Colorímetro "Klett Summerson", com filtro n. 60 .

- Balança analítica Mettler com capacidade de $200 \mathrm{~g}$ e precisão de $0,1 \mathrm{mg}$.

- Agitador mecânico LAB-LINE SUPER MIXER.

- Estufa FABBE.

1.3 - REATIVOS

- Solução de acetato de sódio IN, pH 5,0.

- Solução de cloreto de sódio a $2,5 \%$.

- Peróxido de hidrogênio a $30 \%$.

- Solução de oxalato de potássio a $10 \%$. 
- Solução de ácido oxálico a 9,5\%.

- Fita de magnésio elementar

- Solução de citrato de sódio 0,3 M.

- Solução de bicarbonato de sódio $1 \mathrm{M}$.

- Ditionito de sódio em pó.

- Álcool etílico p.a.

- Solução de nitrato de prata a 5\%.

- Solução aquosa de benzidina.

- Solução de carbonato de sódio a 2,5\%.

\section{2 - Método}

\section{1 - PREPARO DAS AMOSTRAS}

Tomou-se $0,5000 \mathrm{~g}$ de cada um dos minerais usados no teste (Mx, M22, M22a, M20 e M31), usando-se quatro repetições por tratamento.

Cada uma das amostras sofreu um preparo que constou das seguintes fases:

- Moagem.

- Eliminação dos sais solúveis e catíons divalentes trocáveis.

- Eliminação da matéria orgânica e óxido de manganês livre.

- Eliminação dos óxidos de ferro livres. Usaram-se dois métodos: o do H-nascente e o do bicarbonato-citrato-ditionito de sódio.

No tratamento em que não houve remoção dos óxidos de ferro livres, após a eliminação da matéria orgânica e óxido de manganês livre o material seguiu para a fase seguinte, ou seja, eliminação dos cloretos.

Nos casos em que se procedeu a remoção dos óxidos de ferro livres foram feitos tantos tratamentos quantos necessários para a máxima remoção dos mesmos. $\mathrm{E}$ isto se constatava tratando-se o sobrenadante obtido, após o desenvolvimento do método, com a solução de carbonato de sódio a 2,5\%: em havendo a formação de um precipitado ferruginoso o processo era repetido.

O número de tratamentos exigidos por amostra encontra-se indicado no Quadro 1. 
QUADRO 1 - Número de tratamentos exigidos para a máxima remoção dos óxidos de ferro livres das amostras de montmorillonita.

\begin{tabular}{|l|c|c|}
\hline \multirow{2}{*}{ Amostra } & Número de & Tratamentos \\
\cline { 2 - 3 } & H-Nascente & Ditionito \\
\hline Mx & 1 & 1 \\
M22 & 2 & 2 \\
M22a & 2 & 1 \\
M20 & 2 & 2 \\
M31 & 1 & 1 \\
\hline
\end{tabular}

- Eliminação dos cloretos.

- Colocação do material em suspensão em água e separação da fração argila.

- Determinação da concentração.

- Preparo de suspensões a $50 \mathrm{ug} / \mathrm{ml}$.

\section{2 - DESENVOLVIMENTO DA COR}

De cada uma das repetições tomaram-se três alíquotas de $10 \mathrm{ml}$, as quais foram colocadas em três tubos de ensaio, aos quais se adicionaram 1,4 ml da solução aquosa de benzidina, seguindo-se enérgica agitação e um repouso de 48 horas. Ao repouso seguiram-se as leituras das absorbâncias, tomando-se o cuidado de agitar os tubos antes do processamento destas.

A média das três leituras foi usada para representar a repetição.

\section{RESULTADOS E DISCUSSÃO}

\section{1 - Resultados}

Os resultados obtidos para absorbância das diversas repetições de que consistiu o teste encontram-se resumidos nos Quadros 2, 3 e 4. 
QUADRO 2 - Valores obtidos para absorbância das diversas montmorillonitas no tratamento em que não houve eliminação dos óxidos de ferro livres.

\begin{tabular}{|c|c|c|}
\hline \multirow{2}{*}{ Repetição } & Absorbância & \multirow{2}{*}{ Média } \\
\hline & Individual & \\
\hline \multicolumn{3}{|c|}{ Montmorillonita $\mathrm{X}$} \\
\hline 1 & $25-23-21$ & 23 \\
\hline 2 & $19-16-16$ & 17 \\
\hline 3 & $22-19-18$ & 20 \\
\hline 4 & $19-17-18$ & 18 \\
\hline \multicolumn{3}{|c|}{ Montmorillonita 22} \\
\hline 1 & $39-33-32$ & 35 \\
\hline 2 & $37-36-40$ & 38 \\
\hline 3 & $47-47-41$ & 45 \\
\hline 4 & $46-46-44$ & 45 \\
\hline \multicolumn{3}{|c|}{ Montmorillonita 22a } \\
\hline 1 & $21-19-22$ & 21 \\
\hline 2 & $21-21-20$ & 21 \\
\hline 3 & $20-18-19$ & 19 \\
\hline 4 & $15-17-17$ & 16 \\
\hline \multicolumn{3}{|c|}{ Montmorillonita 20} \\
\hline 1 & $41-45-42$ & 43 \\
\hline 2 & $39-37-40$ & 39 \\
\hline 3 & $44-44-43$ & 44 \\
\hline 4 & 39-38-39 & 39 \\
\hline \multicolumn{3}{|c|}{ Montmorillonita 31} \\
\hline 1 & $25-25-26$ & 25 \\
\hline 2 & $27-27-24$ & 26 \\
\hline 3 & 23-23-28 & 25 \\
\hline 4 & $19-18-19$ & 19 \\
\hline
\end{tabular}


QUADRO 3 - Valores obtidos para absorbância das diversas montmorillonitas no tratamento em que os óxidos de ferro livres foram removidos pelo método do $\mathrm{H}$-nascente.

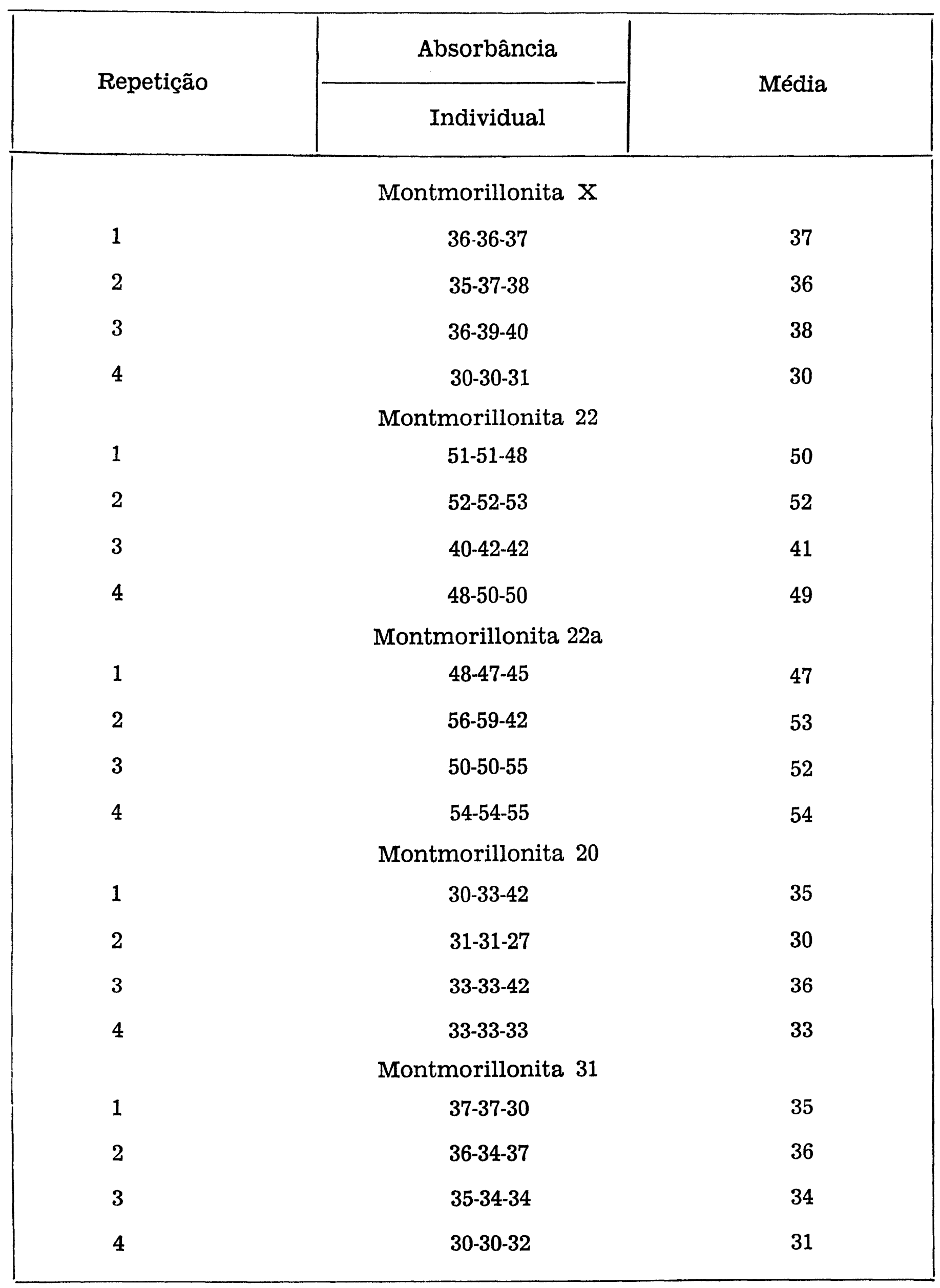


QUADRO 4 - Valores obtidos para absorbância das diversas montmorillonitas no tratamento em que os óxidos de ferro livres foram removidos pelo método do bicarbonato-citrato-ditionito de sódio.

\begin{tabular}{|c|c|c|}
\hline \multirow{2}{*}{ Repetição } & Absorbância & \multirow{2}{*}{ Média } \\
\hline & Individual & \\
\hline \multicolumn{3}{|c|}{ Montmorillonita $\mathrm{X}$} \\
\hline 1 & $19-20-17$ & 19 \\
\hline 2 & $20-20-21$ & 20 \\
\hline 3 & 21-19-20 & 20 \\
\hline 4 & 21-24-19 & 21 \\
\hline \multicolumn{3}{|c|}{ Montmorillonita 22} \\
\hline 1 & $18-18-18$ & 18 \\
\hline 2 & $15-14-23$ & 17 \\
\hline 3 & $16-19-18$ & 18 \\
\hline 4 & $21-21-17$ & 20 \\
\hline \multicolumn{3}{|c|}{ Montmorillonita 22a } \\
\hline 2 & $42-42-49$ & 44 \\
\hline 3 & $38-38-37$ & 38 \\
\hline \multirow{2}{*}{4} & $43-45-45$ & 44 \\
\hline & $41-41-40$ & 41 \\
\hline 1 & \multicolumn{2}{|l|}{ Montmorillonita 20} \\
\hline 2 & $40-40-46$ & 42 \\
\hline 3 & $44-47-41$ & 44 \\
\hline 4 & $40-40-41$ & 40 \\
\hline 1 & $40-48-49$ & 46 \\
\hline \multicolumn{3}{|c|}{ Montmorillonita 31} \\
\hline 2 & $26-26-30$ & 27 \\
\hline 3 & $24-28-26$ & 26 \\
\hline 3 & $21-24-25$ & 23 \\
\hline 4 & $24-24-23$ & 24 \\
\hline
\end{tabular}


Para melhor se poder analisar o efeito da eliminação dos óxidos de ferro livres na uniformização da cor azul obtida pela reação da montmorillonita com a benzina, o Quadro 5 contém as diferenças entre os valores médios obtidos para a absorbância dos diversos tipos de montmorillonita (Mx, M22, M22a, M20 e M31) nos diferentes tratamentos utilizados.

QUADRO 5 - Diferenças entre os valores médios obtidos para absorbância dos diferentes tratamentos da Mx, M22, M22a, M20 e M31.

\begin{tabular}{|c|c|c|c|c|}
\hline \multirow{2}{*}{ Comparaçãc } & \multicolumn{3}{|c|}{ Diferenças } & \multirow{2}{*}{$\operatorname{DM}(* *)$} \\
\hline & $\mathrm{CF}(*)$ & $H(*)$ & $D(*)$ & \\
\hline $\operatorname{Mx} \times \mathrm{M} 22$ & 21,3 & 12,7 & 1,7 & $\mathrm{D}$ \\
\hline$M \times \times M 22 a$ & 0,2 & 16,3 & 21,8 & CF \\
\hline $\mathrm{Mx} \times \mathrm{M} 20$ & 21,8 & 1,8 & 23,0 & $\mathrm{H}$ \\
\hline Mx x M31 & 4,3 & 1,3 & 5,0 & $\mathrm{H}$ \\
\hline M22 x M22a & 21,5 & 3,5 & 23,5 & $\mathrm{H}$ \\
\hline $\mathrm{M} 22 \times \mathrm{M} 20$ & 0,5 & 14,5 & 24,7 & $\mathrm{CF}$ \\
\hline M22 x M31 & 17,0 & 14,0 & 6,7 & $\mathbf{D}$ \\
\hline $\mathrm{M} 22 \mathrm{a} \times \mathrm{M} 20$ & 22,0 & 18,0 & 1,2 & $\mathbf{D}$ \\
\hline M22a $\times$ M31 & 4,5 & 17,5 & 16,8 & CF \\
\hline M20 x M31 & 17,5 & 0,5 & 18,0 & $\mathbf{H}$ \\
\hline
\end{tabular}

(*) CF - tratamento em que não houve eliminação dos óxidos de ferro livres.

H - Tratamento no qual os óxidos de ferro livres foram eliminados pelo método de $\mathbf{H}$ -

nascente.

D - tratamento no qual os óxidos de ferro livres foram eliminados pelo método do bicarbonato-citrato-ditionito de sódio.

(**) DM - Tratamento responsável pela diferença mínima de absorbância, quando se comparam duas motmorillonitas diferentes, mas que foram sujeitas ao mesmo tipo de tratamento. 


\section{2 - Análise estatística}

Os resultados obtidos na análise da variância das diversas montmorillonitas nos vários tratamentos encontram-se resumidos nos Quadros 6, 7, 8, 9 e 10. Nos casos em que o teste $F$ foi significativo aplicou-se o teste Tukey (PIMENTEL GOMES, 1966).

\section{1 - MONTMORILLONITA X}

- Teste F

QUADRO 6 - Análise da variância dos diversos tratamentos da $\mathrm{Mx}$.

\begin{tabular}{|l|c|r|r|c|c|}
\hline C. de Variação & G.L. & S.Q. & Q. M. & F & S \\
\hline Tratamentos & 2 & 641,17 & 320,59 & $46,73^{* *}$ & \\
Resíduo & 9 & 61,75 & 6,86 & & 2,62 \\
\hline Total & 11 & 702,92 & & & \\
\hline
\end{tabular}

C. V. $=10,51 \%$

- Teste Tukey $\left.{ }^{1}\right)$

$$
\begin{aligned}
& \hat{\mathbf{Y}}_{1}=\hat{\mathbf{Y}}_{\mathrm{H}}-\hat{\mathbf{Y}}_{\mathrm{S}}=15,75(* *) \\
& \hat{\mathbf{Y}}_{2}=\hat{\mathbf{Y}}_{\mathrm{H}}-\hat{\mathbf{Y}}_{\mathrm{D}}=15,25(* *) \\
& \hat{\mathbf{Y}}_{3}=\hat{\mathbf{Y}}_{\mathrm{D}}-\hat{\mathbf{Y}}_{\mathrm{S}}=0,50
\end{aligned}
$$

Os valores de $\Delta$ (delta), calculados ao nível de 5 e $1 \%$, respectivamente, foram 5,18 e 7,10.

(1) $\hat{\mathbf{Y}}_{1}, \hat{\mathbf{Y}}_{2} \hat{\mathbf{Y}}_{3}-$ estimativas dos contrastes testados.

$\hat{Y}_{S}$ - Eștimativa da média do tratamento em que não houve eliminaçãa dos óxidos de

$\hat{\mathbf{Y}}$ - Estimativa da média do tratamento em que os óxidos de ferro livres foram eliminados pelo método do H-nascente.

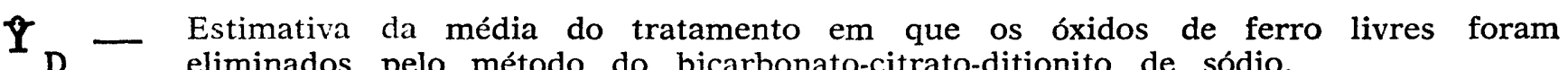
eliminados pelo método do bicarbonato-citrato-ditionito de sódio. 
$2.2-$ MONTMORILLONITA 22

- Teste $\mathrm{F}$

QUADRO 7 - Análise da variância dos diversos tratamentos da M22.

\begin{tabular}{|l|c|r|r|r|r|}
\hline \multicolumn{1}{|c|}{ C. de Variação } & G.L. & S.Q. & Q. M. & F & S \\
\hline Tratamentos & 2 & $1.925,17$ & 962,59 & $57,19 * *$ & \\
Resíduo & 9 & 151,50 & 16,83 & & 4,10 \\
\hline Total & 11 & $2.076,67$ & & & \\
\hline
\end{tabular}

C. V. $=11,49 \%$

- Teste Tukey

Os valores de $\Delta$ (delta), calculados ao nível de 5 e $1 \%$, respectivamente, foram : 8,10 e 11,11 .

$$
\begin{aligned}
& \hat{\mathbf{Y}}_{1}=\hat{\mathbf{Y}}_{\mathrm{H}}-\hat{\mathbf{Y}}_{\mathrm{S}}=7,25 \\
& \hat{\mathbf{Y}}_{2}=\hat{\mathbf{Y}}_{\mathrm{H}}-\hat{\mathbf{Y}}_{\mathrm{D}}=29,75(* *) \\
& \hat{\mathbf{Y}}_{3}=\hat{\mathbf{Y}}_{\mathrm{S}}-\hat{\mathbf{Y}}_{\mathrm{D}}=22,50(* *)
\end{aligned}
$$

2.3 - MONTMORILLONITA 22a

- Teste F

QUADRO 8 - Análise da variância dos diversos tratamentos da M22a.

\begin{tabular}{|l|r|r|r|r|r|}
\hline C. de Variação & G.L. & S.Q. & Q. M. & F & s \\
\hline Tratamentos & 2 & $2.188,50$ & $1.094,25$ & $139,75 * *$ & \\
Resíduo & 9 & 70,50 & 7,83 & & 2,79 \\
\hline Total & 11 & $2.259,00$ & & \\
\hline
\end{tabular}

$$
\text { C. V. }=7,44 \%
$$

- Teste Tukey

Os valores de $\Delta$ (delta), calculados ao nível de 5 e $1 \%$, respectivamente, foram : 5,51 e 7,56.

$$
\begin{aligned}
& \hat{\mathbf{Y}}_{1}=\hat{\mathbf{Y}}_{\mathrm{H}}-\hat{\mathbf{Y}}_{\mathrm{S}}=32,25(* *) \\
& \hat{\mathbf{Y}}_{2}=\hat{\mathbf{Y}}_{\mathrm{H}}-\hat{\mathbf{Y}}_{\mathrm{D}}=9,75(* *) \\
& \hat{\mathbf{Y}}_{3}=\hat{\mathbf{Y}}_{\mathrm{D}}-\hat{\mathbf{Y}}_{\mathrm{S}}=22,50(* *)
\end{aligned}
$$


$2.4-$ MONTMORILLONITA 20

- Teste F

QUADRO 9 - Análise da variância dos diversos tratamentos da M20.

\begin{tabular}{|c|c|c|c|c|c|}
\hline C. de Variação & G.L. & S.Q. & Q. M. & $\mathbf{F}$ & $\mathbf{s}$ \\
\hline Tratamentos & 2 & 204,50 & 102,25 & $14,91 * *$ & \\
\hline Resíduo & 9 & 61,75 & 6,86 & & 2,61 \\
\hline Total & 11 & 266,25 & & & \\
\hline
\end{tabular}

C. V. $=6,65 \%$

- Teste Tukey

Os valores de $\Delta$ (delta), calculados ao nível de 5 e $1 \%$, respectivamente, foram : 5,16 e 7,07.

$$
\begin{aligned}
& \hat{\mathbf{Y}}_{1}=\hat{\mathbf{Y}}_{\mathrm{S}}-\hat{\mathbf{Y}}_{\mathrm{H}}=7,75(* *) \\
& \hat{\mathbf{Y}}_{2}=\hat{\mathbf{Y}}_{\mathrm{D}}-\hat{\mathbf{Y}}_{\mathrm{H}}=9,50(* *) \\
& \hat{\mathbf{Y}}_{3}=\hat{\mathbf{Y}}_{\mathrm{D}}-\hat{\mathbf{Y}}_{\mathrm{S}}=1,75
\end{aligned}
$$

2.5 - MONTMORILLONITA 31

- Teste $F$

QUADRO 10 - Análise da variâncio dos diversos tratamentos da M31.

\begin{tabular}{|l|c|r|rr|r|}
\hline C. de Variação & G.L. & S.Q. & Q. M. & F & S \\
\hline Tratamentos & 2 & 250,17 & 125,09 & $20,57 * *$ & \\
Resíduo & 9 & 54,75 & 6,08 & & 2,46 \\
\hline Total & 11 & 304,92 & & & \\
\hline
\end{tabular}

C. V. $=8,92 \%$

- Teste Tukey

Os valores de $\Delta$ (delta), calculados ao nível de 5 e $1 \%$, respectivamente, foram: 4,86 e 6,67.

$$
\begin{aligned}
& \hat{\mathbf{Y}}_{1}=\hat{\mathbf{Y}}_{\mathrm{H}}-\hat{\mathbf{Y}}_{\mathrm{S}}=10,25\left(^{* *}\right) \\
& \hat{\mathbf{Y}}_{2}=\hat{\mathbf{Y}}_{\mathrm{H}}-\hat{\mathbf{Y}}_{\mathrm{D}}=9,00\left(^{* *}\right) \\
& \hat{\mathbf{Y}}_{3}=\hat{\mathbf{Y}}_{\mathrm{D}}-\hat{\mathbf{Y}}_{\mathrm{S}}=1,25
\end{aligned}
$$


A análise dos resultados obtidos permitiu as seguintes observações :

a) Em todas as montmorillonitas testadas houve influência do tipo de tratamento, sendo o teste $\mathrm{F}$ significativo ao nível de $1 \%$.

b) Nos casos da Mx, M22, M22a e M31 o tratamento com Hnascente foi o que propiciou valores mais elevados para absorbância.

c) No caso da M20, porém, o tratamento em que não houve a eliminação dos óxidos de ferro livres e aquele em que tais óxidos foram eiminados pelo método do bicarbonato-citrato-ditionito de sódio toram significativamente melhores, mas entre eles não se con. seguiu constatar diferenças significativas.

d) Nos casos da Mx, M20 e M31 não se conseguiu constatar diferenças significativas entre o tratamento em que não houve remoção dos óxidos de ferro livres e aquele no qual tais óxidos foram removidos pelo método do bicarbonato-citrato-ditionito de sódio. Já no caso da M22 o tratamento sem remoção dos óxidos de ferro livres foi significativamente melhor do que aquele no qual os referidos óxidos foram eliminados pelo método bicarbonato-citrato-ditionito de sódio, ocorrendo o contrário no caso da M22a.

e) A análise do Quadro 5 revela que, em $70 \%$ dos casos estudados (7 em 10), os tratamentos para elıminação dos óxidos de ferro livres concorreram para maior uniformidade na cor azul do complexo colorido formado, uma vez que determinaram uma diferença menor entre as absorbâncias quando se comparavam duas montmorillonitas diferentes, mas sujeitas ao mesmo tratamento. Nos casos em que seu ef́eito não se fez sentir, as montmorillonitas, em comparação não apresentavam (ou apresentavam em dose muito pequena) diferenças de cor devido à presença de óxidos de ferro livres.

f) Nos casos em que houve influência da eliminação dos óxidos de ferro livres, o método do H-nascente apresentou ligeira vantagem sobre o método do bicarbonato-citrato-ditionito de sódio (4 casos em favor do H-nascente contra 3 em favor do ditionito). Todavia isto parece não ser suficiente para se afirmar que o método do $\mathrm{H}$-nascente seja melhor que o do bicarbonato-citrato-ditionito de sódio no que tange à uniformização da cor azul do complexo resultante da reação da montmorillonita com a benzidina.

A conclusão geral a que se pode chegar após este estudo é que a eliminação dos óxidos de ferro livres parece ser necessária para maior uniformidade da cor azul formada, sendo que, nos casos examinados, o método do H-nascente foi o que aparentou oferecer os me- 
lhores resultados, não apenas no que se refere à uniformização, mas também quanto à intensidade da cor azul formada.

\section{SUMMARY}

COLORIMETRIC STUDY OF MONTMORILLONITE. - Effect of removal of free iron oxides in the montmorillonite benzidine reaction.

The objective of this work is to study the influence of the iron oxide removal in the montmorillonite - benzidine reaction and the influence of the methods used for this removal.

Four montmorillonites references were used and one of unknown origin.

The following tests were conducted:

a) soluble salts, exchangeable cations, organic matter and manganese oxide removal.

b) The same treatment indicated in $\mathbf{a}$ and the iron oxide remoral by the method of JEFFRIES \& JACKSON (1949).

c) The same treatment indicated in a and the iron oxide removal by a dithionite - citrate system buffered with sodium bicarbonate.

There was influence of the type of treatment for all montomorillonites tested.

The iron oxides removal seemed to make uniform the color obtained and the velocity of reaction. The free- $\mathrm{H}$ method gave the best results either for the color uniformization or the intensity of the color.

\section{LITERATURA CITADA}

AGUILLERA, N. H. e M. L. JACKSON, 1953 - Iron oxide removal from soils and clays. Soil Sci. Soc. Am. Proc. 17:359-364.

GRIM, R. E. Clay Mineralogy. 1953 - Mc Graw-Hill Book Company. N.Y. $384 \mathrm{pp}$.

HENDRICKS, S. B. e L. T. ALEXANDER. 1940 - A quantitative test color for the montmorillonite type of clay minerals. Agronomy Journal. Geneva, N.Y. 32:455-457.

JEFRIES, C. D. e M. L. JACKSON. 1949. - Mineralogical analyses of soils. Soil Sci. 68:57-73. 
MEHRA, O. P. e M. L. JACKSON, 1960 - Iron oxide removal and clay by a dithionite-citrate system buffered with sodium bicarbonate. Proc. 7th Natl. Conf. on clays and clays, pp. 317-327. Pergamos Press, N.Y.

PAGE, J. B. 1941. - Unreability of the benzidine color reaction as a test for montmorillonite, Soil Sci. 51:133-140.

PIMENTEL GOMES, F. 1966 - Curso de estatística experimental. 3.. edição. ESALQ. USP, Piracicaba, S.P., Brasil, 404 pp.

SIEGEL, W. 1945. - Detection of montmorillonite With benzidine. In chemical Abstracts. 
